CERN-EP/2000-074

19 April 2000

\title{
A high-resolution tracking hodoscope based on capillary layers filled with liquid scintillator
}

\author{
A. Bay $^{a}$, L. Benussi $^{b}$, N. Bruski ${ }^{c}$, S. Buontempo $^{c}$, C. Currat $^{a}$, N. D'Ambrosio $^{d}$, \\ A.V. Ekimov ${ }^{e}$, A. Ereditato ${ }^{c}$, J.P. Fabre ${ }^{f}$, V. Fanti $^{b}$, D. Frekers ${ }^{d}$, A. Frenkel ${ }^{g}$, \\ S.V. Golovkin ${ }^{e}$, V.N. Govorun ${ }^{e}$, K. Harrison ${ }^{g}$, P. Koppenburg ${ }^{a}$, E.N. Kozarenko ${ }^{h}$, \\ I.E. Kreslo ${ }^{g}$, B. Liberti ${ }^{g}$, G. Martellotti ${ }^{g}$, A.M. Medvedkov ${ }^{e}$, \\ M.R. Mondardini ${ }^{i}$, G. Penso ${ }^{g}$, W.P. Siegmund ${ }^{j}$, V.G. Vasil'chenko ${ }^{e}$, \\ P. Vilain ${ }^{b}$, G. Wilquet ${ }^{b}$, K. Winter ${ }^{i}$, H.J. Wörtche ${ }^{d}$ \\ a Université de Lausanne, Lausanne, Switzerland \\ ${ }^{b}$ IIHE, ULB-VUB, Bruxelles, Belgium \\ c Università "Federico II" and INFN, Napoli, Italy \\ ${ }^{d}$ Westfälische Wilhelms-Universität, Münster, Germany \\ e IHEP, Protvino, Russia \\ $f$ CERN, Genève, Switzerland \\ $g$ Università "La Sapienza" and INFN, Roma, Italy \\ h JINR, Dubna, Russia \\ ${ }^{i}$ Humboldt-Universität, Berlin, Germany \\ j TaperVision, Inc., Pomfret, CT, USA
}

\begin{abstract}
Results are given on tests of a high-resolution tracking hodoscope based on layers of $26-\mu$ m-bore glass capillaries filled with organic liquid scintillator (1-methylnaphthalene doped with R39). The detector prototype consisted of three 2-mm-thick parallel layers, with surface areas of $2.1 \times 21 \mathrm{~cm}^{2}$. The layers had a centre-to-centre spacing of $6 \mathrm{~mm}$, and were read by an optoelectronic chain comprising two electrostatically focused image intensifiers and an Electron-Bombarded Charge-Coupled Device (EBCCD). Tracks of cosmic-ray particles were recorded and analysed. The observed hit density was $6.6 \mathrm{hits} / \mathrm{mm}$ for particles crossing the layers perpendicularly, at a distance of $1 \mathrm{~cm}$ from the capillaries' readout end, and $4.2 \mathrm{hits} / \mathrm{mm}$ for particles at a distance of $20 \mathrm{~cm}$. A track segment reconstructed in a single layer had an rms residual of $\sim 20 \mu \mathrm{m}$, and allowed determination of the track position in a neighbouring layer with a precision of $\sim 170 \mu \mathrm{m}$. This latter value corresponded to an rms angular resolution per layer of about $30 \mathrm{mrad}$. A comparison is made between capillary layers and silicon microstrip planes.
\end{abstract}

PACS: 29.40.Gx; 29.40.Mc; 42.79.Pw; 85.60.Gz

Keywords: Tracking and position-sensitive detectors; Scintillating fibres; Capillaries; Optoelectronics; Image intensifiers; EBCCD

(Submitted to Nuclear Instruments $\&$ Methods A) 


\section{Introduction}

Tracking devices based on glass capillaries filled with liquid scintillator have been extensively studied over the past few years [1]. The principle of operation of the liquid-filled capillaries is the same as for glass and plastic scintillating fibres. Light is emitted when an ionizing particle traverses the liquid scintillator (core material) and a fraction of this light is trapped in the capillary through total internal reflection at the capillary walls (cladding material). The light that propagates in the capillaries forms an image of the particle track, and can be read using an optoelectronic chain. This typically consists of one or more image intensifiers coupled to a photosensitive Charge-Coupled Device (CCD) or to an Electron-Bombarded Charge-Coupled Device (EBCCD).

Glass and plastic scintillating fibres and liquid-filled capillaries (with diameters of 15-30 $\mu \mathrm{m}$ ) have been assembled in coherent bundles, and give a spatial resolution of the order of tens of microns. Small-volume bundles (cross-section of $5 \times 5 \mathrm{~mm}^{2}$ and length of a few centimetres) have been investigated as active targets for studies of beauty production in a fixed-target environment [2]. In a more recent development, large-volume capillary bundles (cross-section of $2 \times 2 \mathrm{~cm}^{2}$ and length of up to $1.8 \mathrm{~m}$ ) have been successfully tested [1] for use in neutrino experiments.

As an alternative to being assembled in bundles, capillaries and fibres can also be assembled in small-thickness layers. Such layers can be used to construct tracking devices with a geometry resembling that of a detector formed of silicon microstrip planes. Layers of large-diameter $(0.5-1.0 \mathrm{~mm})$ plastic scintillating fibres have been used in experiments UA2 [3,4] and WA95-CHORUS [5] at CERN, and a beam-vertex detector based on onefibre-thick layers of $200 \times 200 \mu \mathrm{m}^{2}$ plastic fibres has been operated in experiment E917 [6] at the Brookhaven National Laboratory.

The light yield of specially prepared liquid scintillators [7-9] is sufficiently high that a capillary layer with a thickness of $1-2 \mathrm{~mm}$ and comprising capillaries of $20-30 \mu \mathrm{m}$ bore will measure several points on the trajectory of a traversing particle. In contrast to a silicon microstrip plane, which measures a single track point per particle, a capillary layer will then record track segments, helping to reduce ambiguities in the reconstruction of high-multiplicity interactions. A method for track reconstruction in capillary layers, starting from track segments and using a Kalman-filter technique, has been developed [10] in a study of simulated events. For the situation considered in the present paper, the method has high efficiency if the average number of track points per layer is at least 6-7.

This paper presents results from tests of a detector prototype formed of three capillary layers. The layers had a thickness of $2 \mathrm{~mm}$ and consisted of capillaries having an inner diameter of $26 \mu \mathrm{m}$. Tracks of cosmic-ray particles were recorded, and have been used to evaluate the detector's spatial and angular resolution. Performance characteristics of capillary layers are compared with those of silicon microstrip planes.

The work summarized in this paper has been carried out in the framework of the CERN Research and Development Project RD46 [11].

\section{Construction and filling of capillary layers}

Each of the three layers in the detector prototype was composed of 10 elements, an individual element having square cross-section and comprising 4392 capillaries. These elements were drawn by TaperVision ${ }^{1}$, and the layer assembly was performed at CERN.

\footnotetext{
${ }^{1}$ TaperVision Inc., Pomfret, CT 06258, USA.
} 
A preform, obtained as a tube having a diameter of $36.5 \mathrm{~mm}$ and $1.5-\mathrm{mm}$-thick-walls of Schott $^{2} 8250$ borosilicate glass (refractive index 1.49 , density $2.28 \mathrm{~g} / \mathrm{cm}^{3}$, softening point $720^{\circ} \mathrm{C}$ ), was drawn to produce capillaries with a diameter of $3.8 \mathrm{~mm}$. These were stacked in groups of 61 to form mini-bundles of hexagonal cross-section (Fig 1). Rods of black glass were inserted in about a quarter of the interstices between capillaries, and acted as an extra-mural absorber (EMA) in the finished detector, suppressing crosstalk. Mini-bundles were drawn such that the distance between parallel sides was decreased from $30 \mathrm{~mm}$ to $2.3 \mathrm{~mm}$.

Layer elements were assembled as arrays of $8 \times 9$ mini-bundles (Fig. 1). A final drawing stage then reduced the cross-section of a layer element from $18.3 \times 18.5 \mathrm{~mm}^{2}$ to approximately $2 \times 2 \mathrm{~mm}^{2}$. This drawing stage was performed at relatively low temperature, in order to limit surface tension's reduction of the packing fraction, defined as the ratio between the open area of a layer element and the element's total cross-section. After the final drawing stage, the capillaries' inner and outer diameters were respectively $26 \mu \mathrm{m}$ and $32 \mu \mathrm{m}$, corresponding to a packing fraction of $\sim 67 \%$.

A layer was constructed from a set of 10 elements, glued together in a single row. No surrounding tube was placed about the elements, so that adjacent edges interlocked (Fig. 1). Each layer had transverse dimensions of $2 \times 21 \mathrm{~mm}^{2}$, including the small amount of glue, and was cut to a length of $21 \mathrm{~cm}$.

Capillaries were filled with a liquid scintillator based on 1-methylnaphthalene (1MN, refractive index 1.62 , density $1.0 \mathrm{~g} / \mathrm{cm}^{3}$ ) doped with $3 \mathrm{~g} / \mathrm{l}$ of $\mathrm{R} 39^{3}$. This scintillator emits in the green region, with peak emission at around $500 \mathrm{~nm}$. The liquid scintillator was introduced into the layers through application of an argon overpressure, care being taken to avoid deterioration of the scintillator's light yield through contact with air. The filling procedure has been improved relative to that used previously [1], in that the filling apparatus is more compact and the filling time has been significantly reduced $[12,13]$.

Taking into account the total amounts of glass and liquid scintillator, the radiation length and interaction length for the layer material were respectively $X_{0}=25 \mathrm{~cm}$ and $\lambda_{I}=64 \mathrm{~cm}$.

\section{Setup for tests}

In the test setup, the three capillary layers were placed parallel to one another, with a centre-to-centre spacing of $6 \mathrm{~mm}$, and formed a tracking hodoscope (Fig. 2). The performance of this hodoscope has been studied using cosmic-ray particles. The capillary layers, labelled $\mathrm{L}_{1}, \mathrm{~L}_{2}$ and $\mathrm{L}_{3}$, with $\mathrm{L}_{1}$ uppermost, were read by an optoelectronic readout chain. This consisted of two image intensifiers followed by an EBCCD, all made by Geosphaera. A mechanical system allowed the layers to be pushed against the first image intensifier, so that the liquid scintillator contained in the capillaries came in direct contact with the entrance fibre-optic window of the readout chain.

The first image intensifier, which introduced a magnification of $m=0.62$, had a multialkali photocathode of high quantum efficiency: about $20 \%$ at $\sim 500 \mathrm{~nm}$, the wavelength of maximum sensitivity. It was operated at a voltage of $15 \mathrm{kV}$ and gave a gain of $\sim 25$ photons per photon. The second image intensifier, of magnification $m=1$, had an operating voltage of $7 \mathrm{kV}$ and a gain of $\sim 8$ photons per photon. The EBCCD [14] was essentially a zoomable image intensifier where a reversed, thinned CCD was mounted

\footnotetext{
${ }^{2}$ Schott Glas, 55122 Mainz, Germany.

${ }^{3}$ R39 is a trademark of the Geosphaera Research Centre, Moscow 117261, Russia.
} 
in place of the phosphor screen. Electrons emitted at the photocathode were accelerated through $15 \mathrm{kV}$ towards the CCD, which consisted of $1024 \times 1024$ pixels with areas of $13 \times 13 \mu \mathrm{m}^{2}$. In order that the layers' transverse dimensions should fit on the CCD, the voltage of the zoom electrode was set so as to magnify the image by a factor $m=0.75$. Each capillary then occupied an area at the CCD corresponding to 1.2 pixels. The phosphor screens of the first and second image intensifiers had decay times of about $10 \mu \mathrm{s}$ and $200 \mu$ s respectively. The slow response of the phosphors was adequate for the low-rate tests performed and provided an optical memory, allowing time for a trigger decision.

A cosmic-ray trigger was defined by a coincidence between two plastic scintillators measuring $2 \times 2 \mathrm{~cm}^{2}$. These were placed above and below the three capillary layers, at a variable distance $d$ from their readout end. The trigger occurred about 150 ns after a physical event.

The EBCCD had no fast-clear facility, and the CCD chip was cleared by continuous readout at a pixel clock frequency of $10 \mathrm{MHz}$. When a trigger arrived, the clearing was interrupted and a gate pulse was applied to the EBCCD, enabling transmission of the image to the CCD. The light produced by the phosphor of the second image intensifier was integrated over a time of the order of $1 \mathrm{~ms}$, and was then read in $110 \mathrm{~ms}$ by the acquisition system.

A reference grid, consisting of $20-\mu \mathrm{m}$-width transparent lines, etched at $2 \mathrm{~mm}$ intervals on the surface of a mirror, was positioned against the non-readout end of the capillary layers. The grid was used to check the overall magnification of the readout system, and to evaluate corrections for distortion. The mirror reflected incident light back towards the readout end.

\section{Analysis and results}

When the reference grid was illuminated from behind by an LED, the slight pincushion distortion introduced by the optoelectronic chain was seen in the image recorded by the CCD (Fig. 3a). The distortion was parameterised assuming that the magnification $m$ of the chain varies with the distance $R$ from the optical axis as $m(R)=m_{0}\left(1+\alpha R+\beta R^{2}\right)$, where $m_{0}$ is the magnification on the axis. The parameters $\alpha$ and $\beta$ were determined from a fit to the grid image and distortion effects were corrected for (Fig. 3b). The data for the reference grid were additionally used to define the region occupied at the CCD by each of the capillary layers.

Data for cosmic-ray particles were collected with the trigger counters at several distances from the capillaries' readout ends, with around 300 particle tracks recorded at each distance. During data taking, 8 background frames were read every 10 minutes (EBCCD gate closed), then the average pixel pulse height over the 8 frames was adopted as a measure of the dark-current background. Cosmic-ray events were recorded after background subtraction, with suppression of pixels having a pulse height below 30 ADC channels on a scale extending to 256. Track images for particles in triggered events (including some examples of interactions, not used in the subsequent analysis), were clearly seen in the three capillary layers (Fig. 4).

A track analysis was performed. Taking into consideration only the regions of the CCD corresponding to the capillary layers, clusters were obtained by grouping together adjacent pixels with pulse height above a threshold value set, after tuning, to $60 \mathrm{ADC}$ channels. A cluster's centroid was calculated by averaging over the coordinates of the 
Table 1: Root-mean-square track residuals for track fits relative to individual capillary layers and relative to all three layers, as a function of the distance $d$ between track crossing point and readout surface.

track residual $(\mu \mathrm{m})$

\begin{tabular}{ccccc}
\cline { 2 - 4 }$d(\mathrm{~cm})$ & layer $\mathrm{L}_{1}$ & layer $\mathrm{L}_{2}$ & layer $\mathrm{L}_{3}$ & all layers \\
\hline 1 & $30 \pm 3$ & $29 \pm 3$ & $26 \pm 3$ & $62 \pm 2$ \\
3 & $25 \pm 3$ & $25 \pm 3$ & $21 \pm 2$ & $55 \pm 2$ \\
5 & $22 \pm 2$ & $21 \pm 2$ & $20 \pm 2$ & $50 \pm 1$ \\
9 & $21 \pm 2$ & $19 \pm 2$ & $21 \pm 2$ & $50 \pm 1$ \\
15 & $22 \pm 2$ & $23 \pm 2$ & $21 \pm 2$ & $48 \pm 1$ \\
20 & $22 \pm 2$ & $27 \pm 3$ & $23 \pm 2$ & $60 \pm 2$
\end{tabular}

cluster's pixels. In forming the average, pixel coordinates were corrected for the magnification and distortion of the readout system, and were weighted by the pixel pulse height. Sequences of approximately aligned clusters were identified, and straight lines were fitted to their centroids. These track fits were performed both for individual layers, with a requirement of at least 4 clusters in the layer considered, and for the three layers together, when a minimum of 15 clusters was required.

The gains of the optoelectronic chain and of the readout electronics were kept at relatively high values so as to avoid losing hits (electrons emitted at the photocathode of the first image intensifier and detected at the CCD). The high overall gain resulted in some merging of signals from hits that were close together, and so the number of hits per cluster used in the track reconstruction was sometimes greater than 1 . The average number of hits per cluster has been estimated both through a statistical analysis of cluster pulse heights and through a visual examination of track images, and has been found to be $\sim 1.6$.

Hit densities for the three capillary layers decreased from 6.6 hits $/ \mathrm{mm}$ to $4.2 \mathrm{hits} / \mathrm{mm}$ as the distance $d$ between the trigger counters and the capillaries' readout ends was increased from $1 \mathrm{~cm}$ to $20 \mathrm{~cm}$ (Fig. 5). A single-exponential fit to the data on hit density as a function of $d$ yields a light-attenuation length of $48 \pm 9 \mathrm{~cm}$, in good agreement with the short-distance attenuation measured for capillaries assembled in large-volume bundles [1].

Distributions of hit displacements from fitted tracks (track residuals) have been obtained both for track fits relative to a single capillary layer (Fig. 6a, 6b, 6c) and for the track fits relative to all three layers (Fig. 6d). Values for the root-mean-square (rms) track residual $\sigma_{t r}$ have been calculated ${ }^{4}$ and are reported in Table 1.

The spatial resolution achieved with an individual layer depends on physical effects (delta-ray production and Coulomb scattering), on the capillary geometry, on the layer uniformity, and on the resolution and distortion of the optoelectronic chain. The spatial resolution for the three layers together is additionally sensitive to inhomogeneities and misalignments of the layers. For $5 \mathrm{~cm} \leq d \leq 20 \mathrm{~cm}$ the individual layers were characterised by $\sigma_{t r} \simeq 20 \mu \mathrm{m}$, compared with $\sigma_{t r} \simeq 30 \mu \mathrm{m}$ for capillaries in large-volume bundles [1], whereas the three layers together had $\sigma_{t r} \simeq 50 \mu \mathrm{m}$. For smaller $d$ values $(d<5 \mathrm{~cm})$, the diffuse crosstalk light encountered the EMA with lower probability and caused $\sigma_{t r}$ to increase.

\footnotetext{
${ }^{4}$ In this paper, the root-mean-square of a distribution is evaluated as the full width at half maximum divided by $2 \sqrt{2 \ln 2}$.
} 
To estimate the angular resolution obtained with the detector prototype, tracks reconstructed in individual layers have been compared. For each event, tracks fitted in $\mathrm{L}_{1}$ and tracks fitted in $\mathrm{L}_{3}$ were extrapolated to $\mathrm{L}_{2}$, and the coordinates $x_{1}$ and $x_{3}$ of their intersections with a reference axis, at the mid-height of $\mathrm{L}_{2}$, were calculated. The coordinate $x_{2}$ at which the reference axis was crossed by the track fitted in $\mathrm{L}_{2}$ was also determined, then the differences $\delta_{12}=x_{1}-x_{2}$ and $\delta_{32}=x_{3}-x_{2}$ were computed. The distributions of $\delta_{12}$ and $\delta_{32}$ for different distances $d$ did not vary significantly. Their mean values were contained in an interval of $\sim \pm 50 \mu \mathrm{m}$, which is consistent with the layers being aligned to better than $0.5 \mathrm{mrad}$. Summed over all distances $d, \delta_{12}$ and $\delta_{32}$ had the distributions shown in Fig. 7, with rms values of $167 \pm 7 \mu \mathrm{m}$ and $176 \pm 6 \mu \mathrm{m}$ respectively. Recalling that the centre-to-centre spacing of the layers was $6 \mathrm{~mm}$, these values imply that the rms angular resolution for a track reconstructed using a single capillary layer was about $30 \mathrm{mrad}$. For perfect layer geometry (no inhomogeneities), a Monte-Carlo calculation indicates that the angular resolution would be improved to $\sim 20 \mathrm{mrad}$.

\section{Comparison between capillary layers and silicon microstrip planes}

A detector based on capillary layers is in some ways similar to a detector formed of silicon microstrip planes. Both types of device consist of an arrangement of small-thickness modules, and both can be used as microvertex detectors for studies of the production and decay of short-lived particles, including charmed hadrons, beauty hadrons and tau leptons. Differences between the performance characteristics of the two kinds of modules can determine which is the more suitable for a given application. These differences are discussed below, with consideration of capillary layers of the type tested - layers having a thickness of $2 \mathrm{~mm}$, corresponding to $8.1 \times 10^{-3}$ radiation lengths - and silicon planes having a thickness of $300 \mu \mathrm{m}$, a value often used in practice [15-20] and equivalent to $3.2 \times 10^{-3}$ radiation lengths.

A 2-mm-thick capillary layer records an average of about 10 hits along the track of a traversing charged particle at perpendicular incidence. In an experiment, track segments would be reconstructed in each individual layer and track segments from different layers would be matched for both position and direction. This can be of great help for pattern recognition, especially in the case of high-multiplicity events. Using a single layer, the rms track residual is $\sim 20 \mu \mathrm{m}$ and, for the level of homogeneity currently achieved in layer construction, track angles are determined with an rms error of $\sim 30$ mrad.

A 300- $\mu$ m-thick silicon microstrip plane generally has a detection efficiency of between 0.90 and 0.98 [15-20]. For ideal efficiency, the number of measured points per traversing charged particle is 1 (or 2 for double-sided readout), so that the number of measured points per radiation length is lower than with capillaries by a factor of 4 (or 2 for doublesided readout). For pitch values between $10 \mu \mathrm{m}$ and $60 \mu \mathrm{m}$, silicon microstrip planes give an rms track residual in the range 2-18 $\mu \mathrm{m}$ [15-20] (approximately $1 / \sqrt{12}$ times the pitch), and so the point-measurement precision is better for silicon planes than for the capillary layers. However, the higher number of hits per radiation length using capillaries means that the two types of detector allow a similar precision in the determination of track parameters.

Capillary readout can be performed using optoelectronic components, which allow a high degree of multiplexing. For example, an optoelectronic chain based on one or more image intensifiers and a megapixel EBCCD allows readout of several capillary layers, 
comprising $10^{5}-10^{6}$ capillaries. Such a system has the advantage of a low cost per channel, but with the disadvantage that the readout time is long $(\sim 100 \mathrm{~ms})$. Use of several CCDs operated in parallel, with multiple output registers, can significantly decrease the readout time. Alternative approaches, based on fast pixel photodetectors [12] or a highspeed gateable image pipeline [21], have also been studied for high rate experiments. A different situation is encountered with silicon detectors, where each microstrip requires a separate readout channel. This results in a relatively high cost per channel, but the readout is accomplished in a short time (of the order of tens of nanoseconds) with large numbers of channels read in parallel.

A detector based on capillary layers has a greater radiation resistance than a detector formed of silicon microstrip planes. Tests for experiments to be installed at the Large Hadron Collider (LHC) at CERN indicate that a silicon microstrip plane and the associated readout electronics can give a satisfactory performance for doses of up to about 0.1 MGy, provided that the detector is operated under special conditions (for example, at a temperature below $\left.0{ }^{\circ} \mathrm{C}\right)[22,23]$. In contrast, capillaries filled with an appropriate liquid scintillator are able to tolerate dose levels as high as 2 MGy. Measurements [24] for capillaries filled using 1MN doped with R39 show that a dose of $\sim 2$ MGy decreases the scintillation yield by only about $30 \%$ and reduces the attenuation length in the scintillator by a factor of 2-3 (not serious for the propagation distances relevant to capillary layers). In an experiment, the use of a radiation-resistant image guide as a coupling component would allow the readout electronics (tolerated dose of the order of $0.01 \mathrm{MGy}$ ) to be placed at some distance from the capillary layers, in a region where the radiation levels were acceptable. This separation of detector and readout system is generally not possible for a silicon plane.

The surface area of a silicon microstrip plane is usually limited to not much greater than $5 \times 5 \mathrm{~cm}^{2}$. Capillary layers with detection surfaces considerably larger than this can be assembled by taking long capillaries (bundles of capillaries with lengths of $1.8 \mathrm{~m}$ have been successfully used for particle tracking [1]) and by gluing together a high number of layer elements.

Given the measured hit densities, capillary layers having a thickness smaller than $2 \mathrm{~mm}$ can also be constructed. A capillary layer with a depth of $1 \mathrm{~mm}$ would represent approximately the same amount of radiating material as a $300-\mu \mathrm{m}$-thick silicon plane, and would record an average of 5 hits per charged particle traversing the layer perpendicularly. This would result in limited angular information, but the particle-detection efficiency would remain high (probability of $99 \%$ to record $\geq 1$ hit).

\section{Conclusions}

A high-resolution tracking hodoscope comprising three layers of 26 - $\mu \mathrm{m}$-bore glass capillaries filled with organic liquid scintillator (1-MN doped with R39) has been tested. The layers had a thickness of $2 \mathrm{~mm}$, a surface area of $2.1 \times 21 \mathrm{~cm}^{2}$, and a centre-to-centre spacing of $6 \mathrm{~mm}$. Tracks of cosmic-ray particles have been recorded, with capillary readout by an optoelectronic chain consisting of two electrostatically focused image intensifiers and an EBCCD. The observed hit density was $6.6 \mathrm{hits} / \mathrm{mm}$ for particles crossing the layers at a distance of $1 \mathrm{~cm}$ from the capillaries' readout end, and $4.2 \mathrm{hits} / \mathrm{mm}$ for particles at a distance of $20 \mathrm{~cm}$. A single-exponential fit to data on hit density as a function of distance gave the light-attenuation length in the capillaries to be $48 \pm 9 \mathrm{~cm}$. A track segment reconstructed in a single layer had an rms residual of $\sim 20 \mu \mathrm{m}$, and allowed determination 
of the track position in a neighbouring layer with a precision of $\sim 170 \mu \mathrm{m}$, corresponding to an rms angular resolution per layer of about $30 \mathrm{mrad}$. As compared with a typical $300-\mu$ m-thick silicon microstrip plane, the main characteristics of a capillary layer having a thickness of $1-2 \mathrm{~mm}(1.3-2.5$ times the radiating material of the silicon plane) are: it records track segments (position and direction) rather than a single track point per particle, improving pattern recognition; it gives a higher number of hits per radiation length; it has a slightly worse single-hit spatial resolution, but allows similar precision in track reconstruction; its readout time is long $(\sim 100 \mathrm{~ms})$ with the most cost-effective readout schemes, but a much shorter readout time is also possible; it has a much higher radiation resistance (dose levels of up to 2 MGy tolerated); it can have a larger detection

surface. With these characteristics, capillary layers match requirements for track and vertex detectors in a wide variety of experiments.

\section{Acknowledgements}

This work has been carried out in the framework of the CERN Research and Development programme RD46. Finacial support was provided through the European Union's TMR Network Contract ERBFMRX-CT98-0196.

\section{References}

[1] P. Annis et al., Preprint CERN-EP/99-132 (1999), to be published in Nucl. Instr. and Meth. A, and references therein.

[2] C. Angelini et al., Nucl. Instr. and Meth. A289 (1990) 342.

[3] R. E. Ansorge et al., Nucl. Instr. and Meth. A273 (1988) 826.

[4] D.R. Wood, Nucl. Instr. and Meth. A289 (1990) 331.

[5] P. Annis et al., Nucl. Instr. and Meth. A409 (1998) 629.

[6] B.B. Beck et al., Nucl. Instr. and Meth. A412 (1998) 191.

[7] V.G. Vasil'chenko et al., Instr. and Exp. Tech. 40 (1997) 175.

[8] S. Buontempo et al., Nucl. Instr. and Meth. A425 (1999) 494.

[9] G.I. Britvich et al., Nucl. Instr. and Meth. A425 (1999) 498.

[10] P. Koppenburg, Diploma Thesis, Université de Lausanne, 1997.

[11] R. van Dantzig et al., RD46 Proposal, CERN/LHCC 95-7, P60/LDRB, 1995.

[12] J. Konijn et al., Nucl. Instr. and Meth. A418 (1998) 186.

[13] C. Currat, Thesis, Université de Lausanne, in preparation.

[14] S. Buontempo et al., Nucl. Instr. and Meth. A413 (1998) 255.

[15] M. Adinolfi et al., Nucl. Instr. and Meth. A329 (1993) 117.

[16] D. Amidei et al., Nucl. Instr. and Meth. A350 (1994) 73. 
[17] M. Acciarri et al., Nucl. Instr. and Meth. A360 (1995) 103.

[18] V. Chabaud et al., Nucl. Instr. and Meth. A368 (1996) 314.

[19] B. Mours et al., Nucl. Instr. and Meth. A379 (1996) 101.

[20] S. Anderson et al., Nucl. Instr. and Meth. A403 (1998) 326.

[21] A.G. Berkovski et al., Nucl. Instr. and Meth. A380 (1996) 537.

[22] M. Angarano et al., Nucl. Instr. and Meth. A434 (1999) 313.

[23] L. Andricek et al., Nucl. Instr. and Meth. A439 (2000) 427.

[24] S.V. Golovkin et al., Nucl. Instr. and Meth. A362 (1995) 283. 
a)

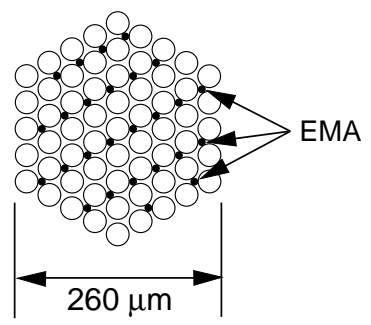

b)

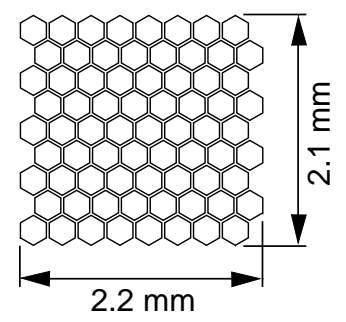

d)

c)

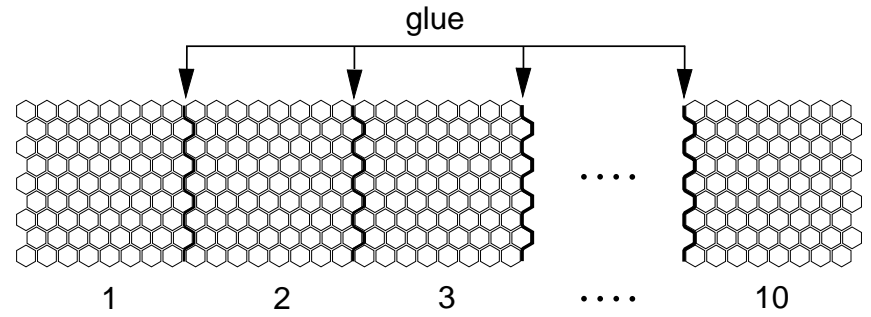

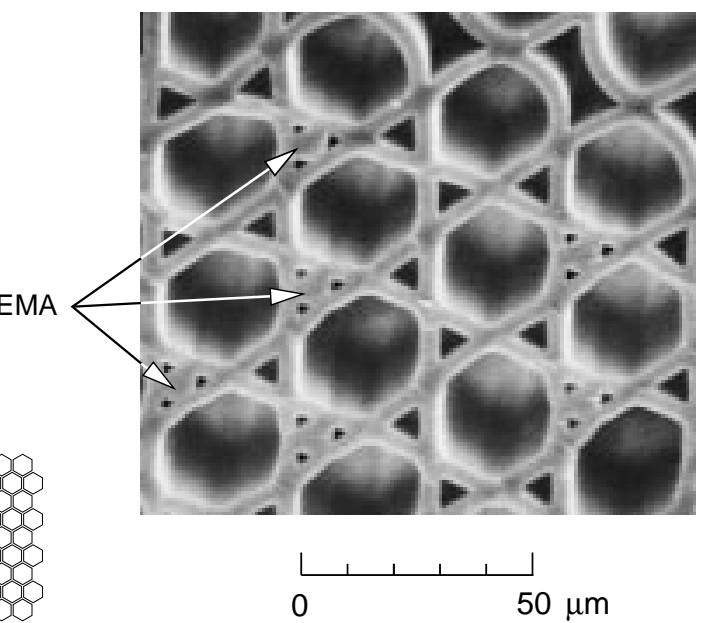

$50 \mu \mathrm{m}$

Figure 1: Cross-sectional views illustrating layer construction: a) a mini-bundle of 61 capillaries, with EMA present in a fraction of the inter-capillary spaces; b) a layer element, consisting of $8 \times 9$ mini-bundles; c) a capillary layer, formed by gluing together 10 elements; d) detail of a mini-bundle, photographed through an electron microscope.

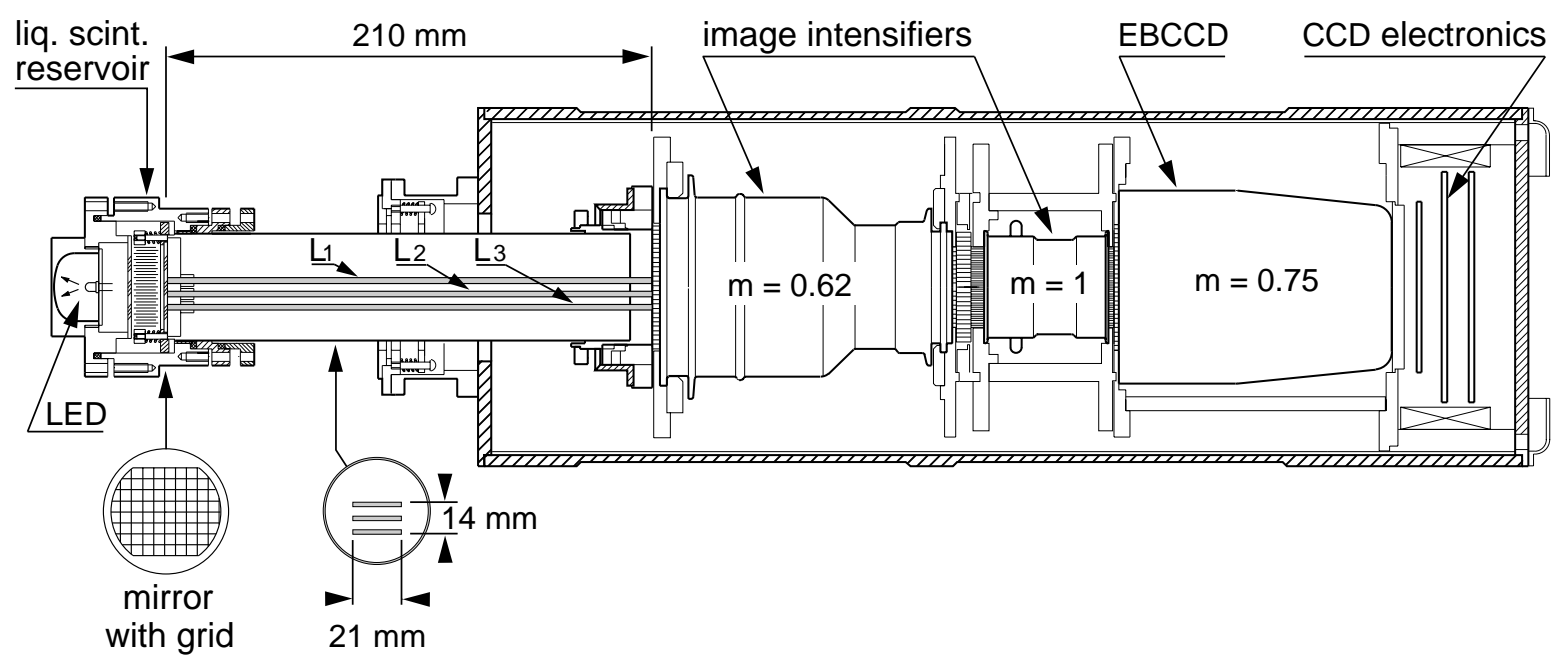

Figure 2: The three capillary layers $\left(\mathrm{L}_{1}, \mathrm{~L}_{2}, \mathrm{~L}_{3}\right)$ and their optoelectronic readout system, comprising two first-generation image intensifiers and a megapixel EBCCD. The magnification $m$ of each component is indicated. A reference grid, placed at the non-readout end of the capillaries, could be illuminated from behind by an LED. 
a)

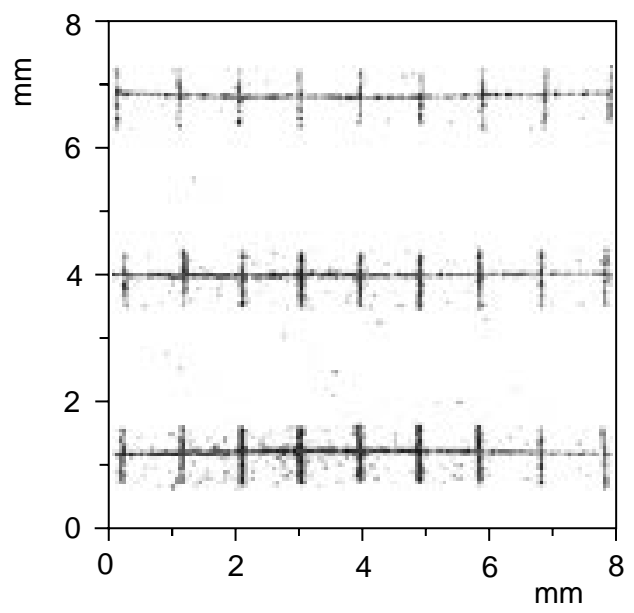

b)

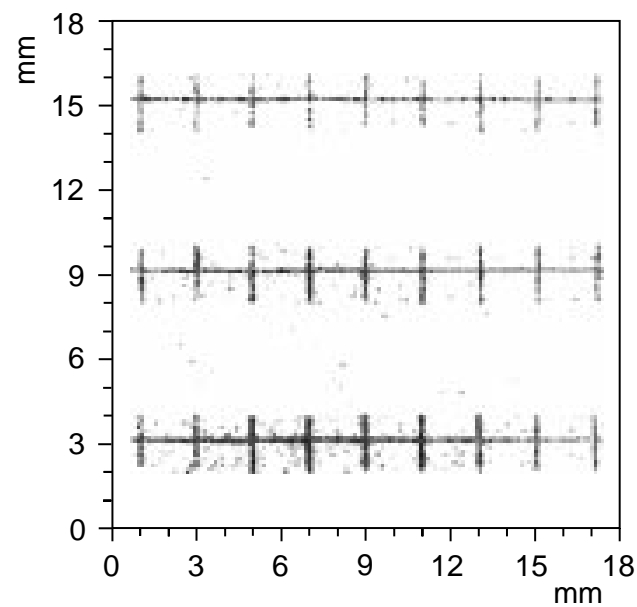

Figure 3: Removal of pincushion distortion introduced by the optoelectronic readout system: a) image of the reference grid as recorded by the EBCCD; b) outline of the grid reconstructed in the space of the capillary layers, after applying the corrections described in the text.

a)

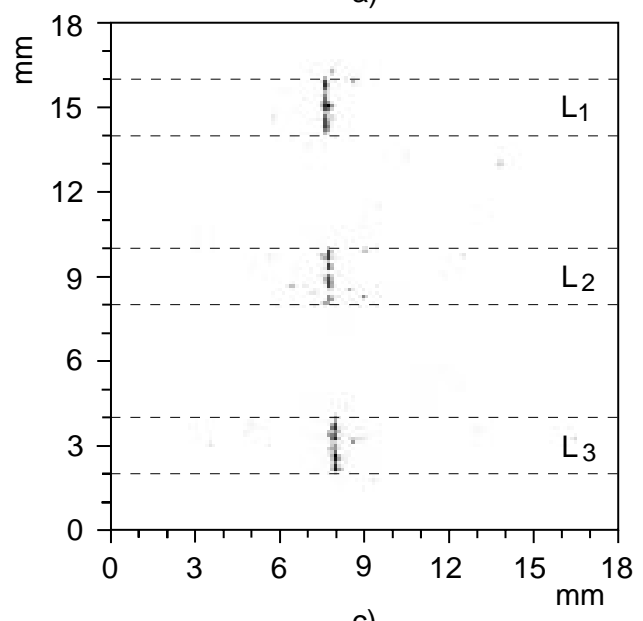

c)

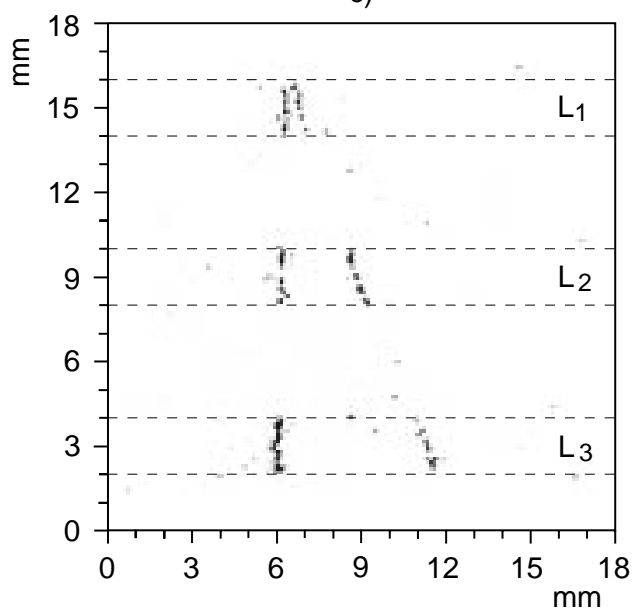

b)

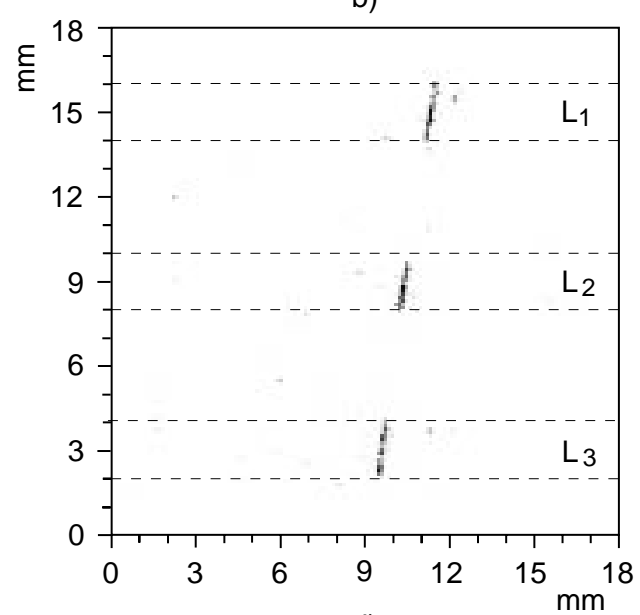

d)

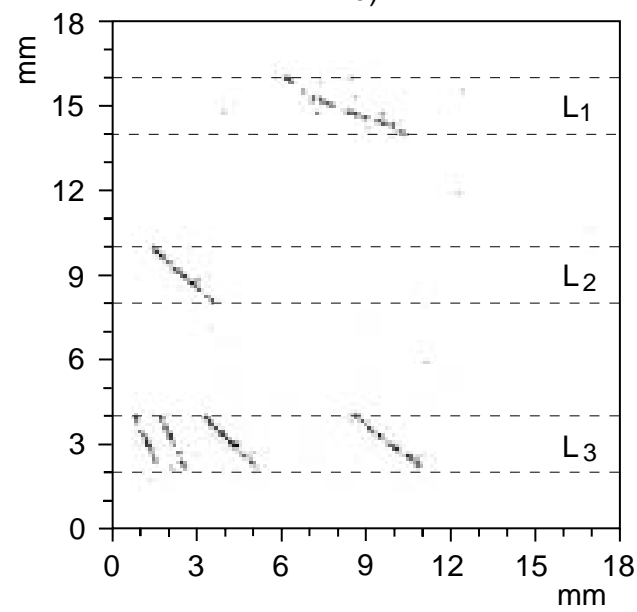

Figure 4: Examples of cosmic-ray events recorded in the capillary layers: a) and b) single-particle events; c) and d) interaction events, not used in the analysis. Dashed lines delimit the regions corresponding to the three layers $\mathrm{L}_{1}, \mathrm{~L}_{2}$ and $\mathrm{L}_{3}$. 


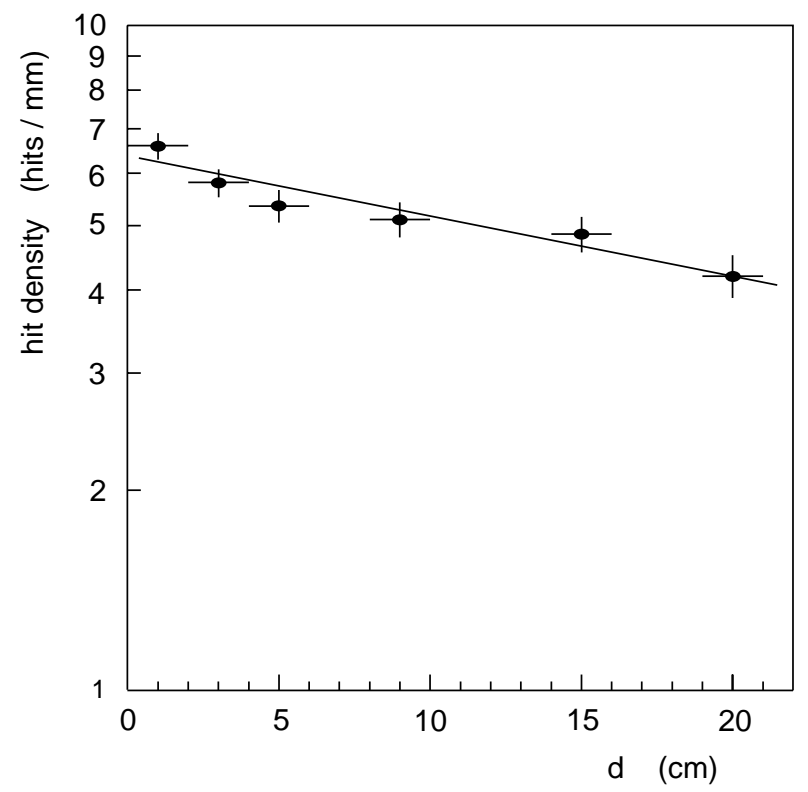

Figure 5: Measured hit density as a function of the track distance $d$ from the readout end of the capillary layers. An exponential fit of the data points is shown, and corresponds to a light-attenuation length in the capillaries of $48 \pm 9 \mathrm{~cm}$. 

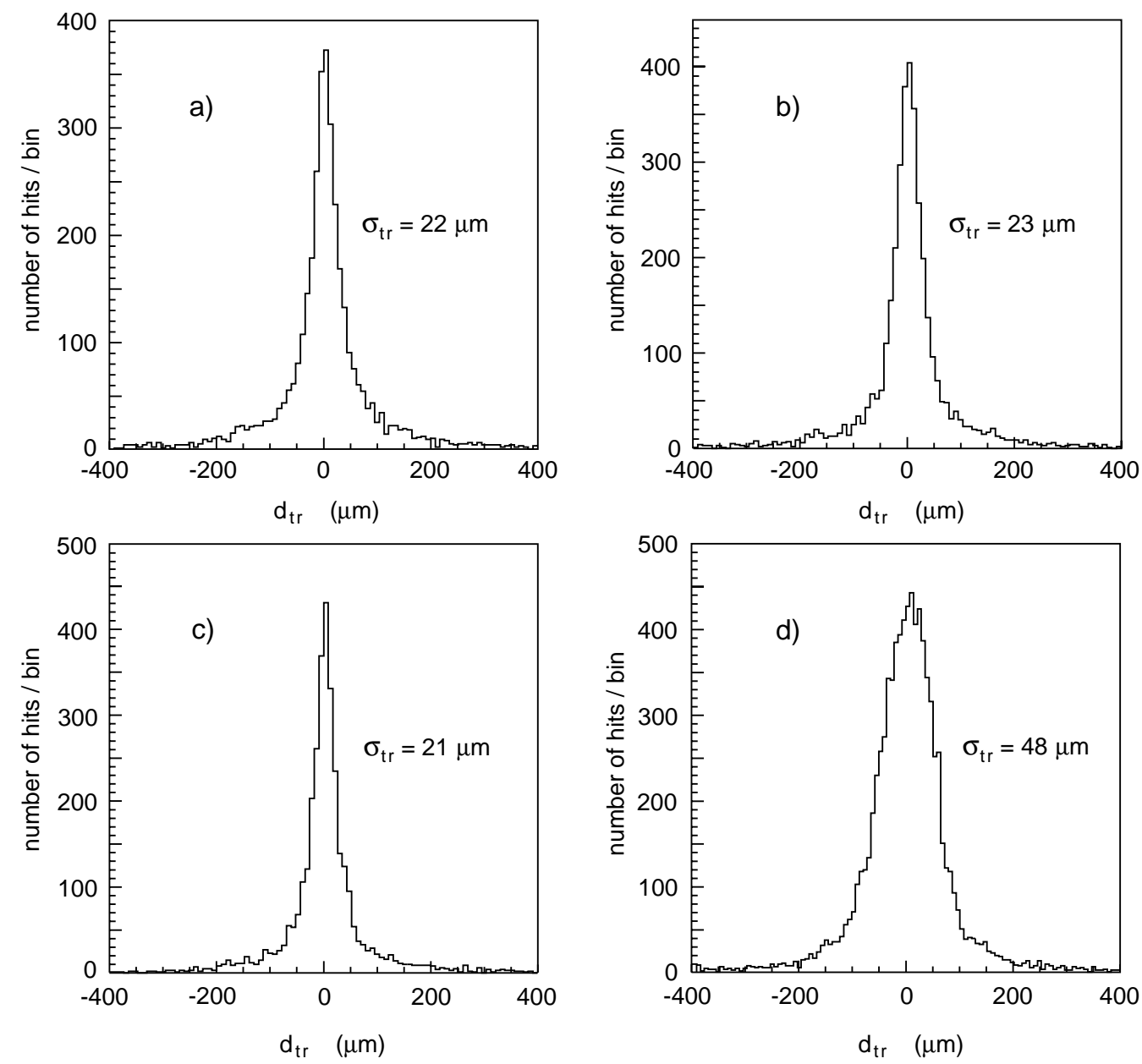

Figure 6: Distribution of track residual $d_{t r}$ for tracks fitted in: a) layer $\mathrm{L}_{1}$ only; b) layer $\mathrm{L}_{2}$ only; c) layer $\mathrm{L}_{3}$ only; d) all layers. Data shown are for tracks crossing the layers at a distance $d=15 \mathrm{~cm}$ from the readout surface. Similar distributions were obtained for other distances $d$, with root-mean-square values as reported in Table 1. 

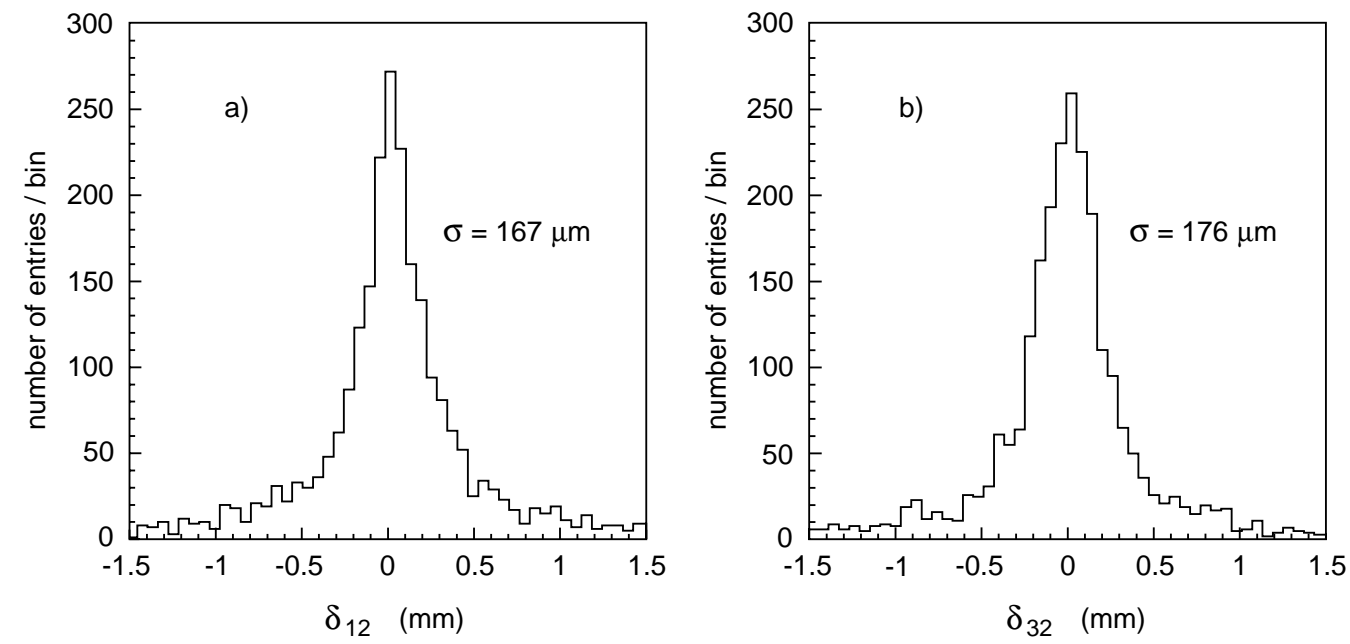

Figure 7: Distribution of: a) distance $\delta_{12}$ between track position measured in layer $\mathrm{L}_{2}$ and position expected from extrapolation of track fitted in layer $\mathrm{L}_{1}$; b) distance $\delta_{32}$ between track position measured in layer $\mathrm{L}_{2}$ and position expected from extrapolation of track fitted in layer $\mathrm{L}_{3}$. Data for tracks crossing the layers at different distances from the readout surface have been summed. 